\title{
Pattern Profile Improvement of Negative Chemical Amplification Resist by Acid Trap Reagent Soaking for $\mathrm{KrF}$ Excimer Laser Lithography
}

\author{
Takashi KASUGA, Yoichi TOMO and Toshiro TSUMORI \\ ULSI Research and Development Group, SONY Corp. \\ 4-14-1 Asahi-cho, Atsugi-shi, Kanagawa, 243 Japan
}

We present a novel pattern profile improvement method for chemically amplified negative photoresist. Based on the mechanism of chemical amplification systems, direct amending of the "acid latent image" prior to post exposure baking can bring drastic pattern profile improvement by using acid trap reagent soaking on the resist surface. We concluded that the acid trapping method combined with highly absorbant chemically amplified resist is one of the promising ways in $\mathrm{KrF}$ excimer lithography.

\section{Introduction}

Since the beginning of research on $\mathrm{KrF}$ excimer laser lithography, the chemically amplified resist systems ${ }^{1}$ ) have been considered to be the most promising methods because they provide high transmittance and high sensitivity at $248 \mathrm{~nm}$ exposure. Various materials with high transparency have been proposed. Some of them, such as XP8843 2) have shown excellent patterning performances on the flat substrates compared with conventional naphtoquinonediazide systems (Fig-1).

However, in the fabrication of the actual device with such a high transparent photoresist, CD variation caused by standing wave phenomenon is one of the very serious problems. This $C D$ variation occurs because of the high reflectivity of substrates in the shorter wavelength region. Fig-2 and Fig-3 show examples of the standing wave phenomenon of XP8843 on device structures. In spite of XP8843's steep profiles the pattern size shifts caused by the standing wave phenomenon are very large. This result shows that XP8843 is not acceptable to be applied in device fabrication without any additional process, eg. the anti reflective technique. 
The employment of highly absorbant resist, so called dyed resist is one of the typical solutions to reduce the standing wave phenomenon. However, the degradation of steep pattern profiles which have been achieved by transparent materials can not be avoided. This is apparently against the initial target of excimer photoresists, high transparency.

In this paper, we propose a novel process for chemically amplified $\mathrm{KrF}$ excimer resist based on the mechanism of acid catalyzed systems 3 ). We employed a new concept for pattern profile improvement, the acid trap method. This method yields satisfying pattern profiles and standing wave characteristics combined with highly absorbant resist.

\section{Method}

\section{Concept}

Fig-4 shows the concept of our acid trap method. As well known, acid catalyzed systems go through the "acid latent image" stage which corresponds to the optical image inside the photoresist film after deep UV irradiation. This stage is unstable because an insoluble region has not been formed until baking process which promotes catalytic chain reactions. In the case of highly absorbant photoresist, the "acid latent image" was degenerated. We focused our work on this stage and tried to improve this degenerated "acid latent image" directly by treating the surface with an "acid trap reagent". We expected, that if we soak the resist surface with an acid trap reagent such as a weak base which does not solve alkaline soluble resin, it would be possible to change the pattern profiles of chemically amplified resists. Imidazole (Fig-5), cyclic amine, which is soluble with water and penetrable into photo resist was employed as an acid trap reagent. Combined with a highly absorbant chemically amplified resist, this process could be very practical for the actual device fabrication.

\section{Process and optimization}

Fig- 6 shows the process flow of this method. In this experiment, commercially available chemically amplified negative photoresist, SAL601-ER7 ${ }^{4}$ )(Abs. 0.92/ $\mu \mathrm{m}$ ) was employed. Before exposure, the resist film was soaked in imidazole aqueous solution of various concentrations and rinsed with freshwater. XP8843(Abs. 0.24/ $\mu \mathrm{m}$ ), highly transparent chemically amplified resist, was used as reference. Exposure was performed with $\mathrm{KrF}$ excimer laser stepper (NA 0.42). All conditions except the imidazole treatment are summarized in Fig-6. The development was not modified.

The soaking was optimized using the cross sectional view of pattern profiles. Fig-7 shows relationships among the resist sidewall angle of $0.35 \mu \mathrm{m} \mathrm{L} / \mathrm{S}$, the resist remaining thickness and the imidazole concentration. These results indicate that it is possible to control the resist profile by changing the imidazole concentration and the soaking time. As a result, optimal conditions (L/S ; $0.2 \mathrm{~N} / 5 \mathrm{~min}, \mathrm{C} / \mathrm{H} ; 0.3 \mathrm{~N} / 10 \mathrm{~min}$ ) were fixed. 


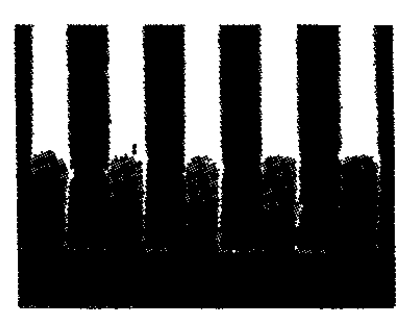

$0.35 \mu \mathrm{mL} / \mathrm{S}$

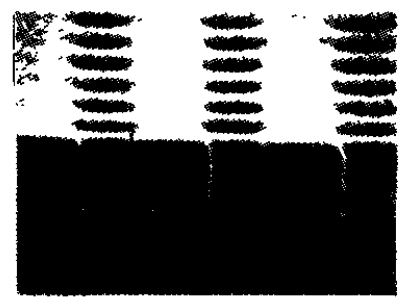

$0.40 \mu \mathrm{mC} / \mathrm{H}$

Exposure Tool NSR1505EX(NA 0.42)

Photoresist Shipley XP8843(SNR248) $1.0 \mu \mathrm{m}$ Abs. $0.20(/ \mu \mathrm{m})$

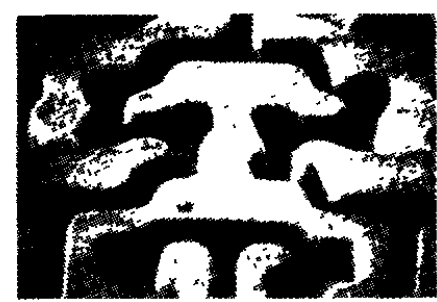

$0.35 \mu \mathrm{m}$ Trench Isolation (After Dry Etching)

Fig-1. XP8843 resist images on the flat Si wafers

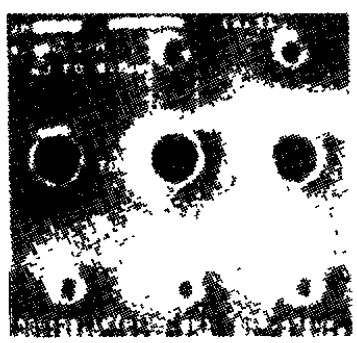

\section{4um Contact Hole}

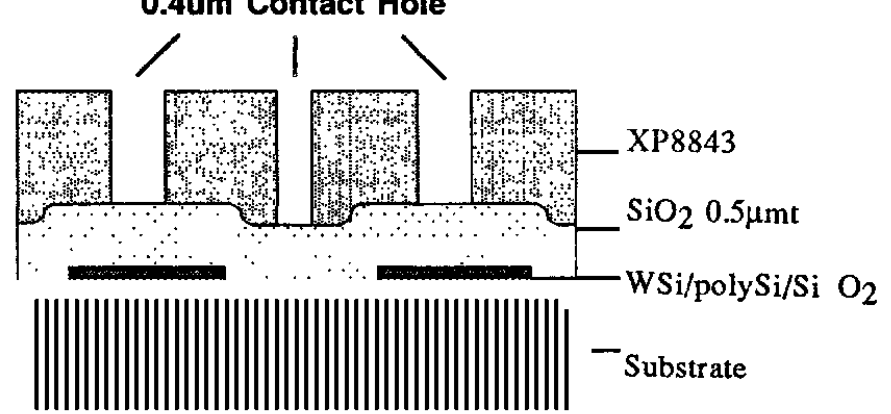

Fig-3. CD variation of XP8843 on the topology; contact holes<smiles>c1c[nH]cn1</smiles>

Fig-5. Acid trap reagent; imidazole

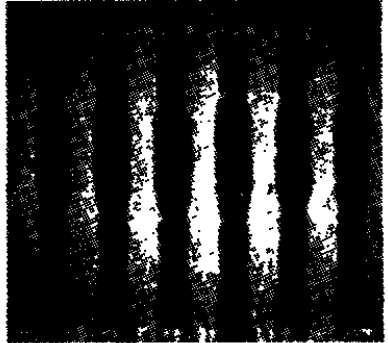

$0.35 \mu \mathrm{m} \mathrm{LS}$

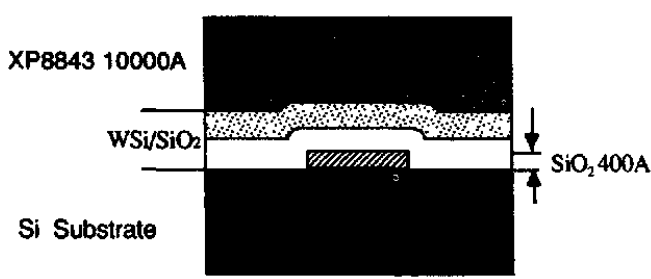

Fig-2. CD variation of XP8843 on the topology; lines and spaces

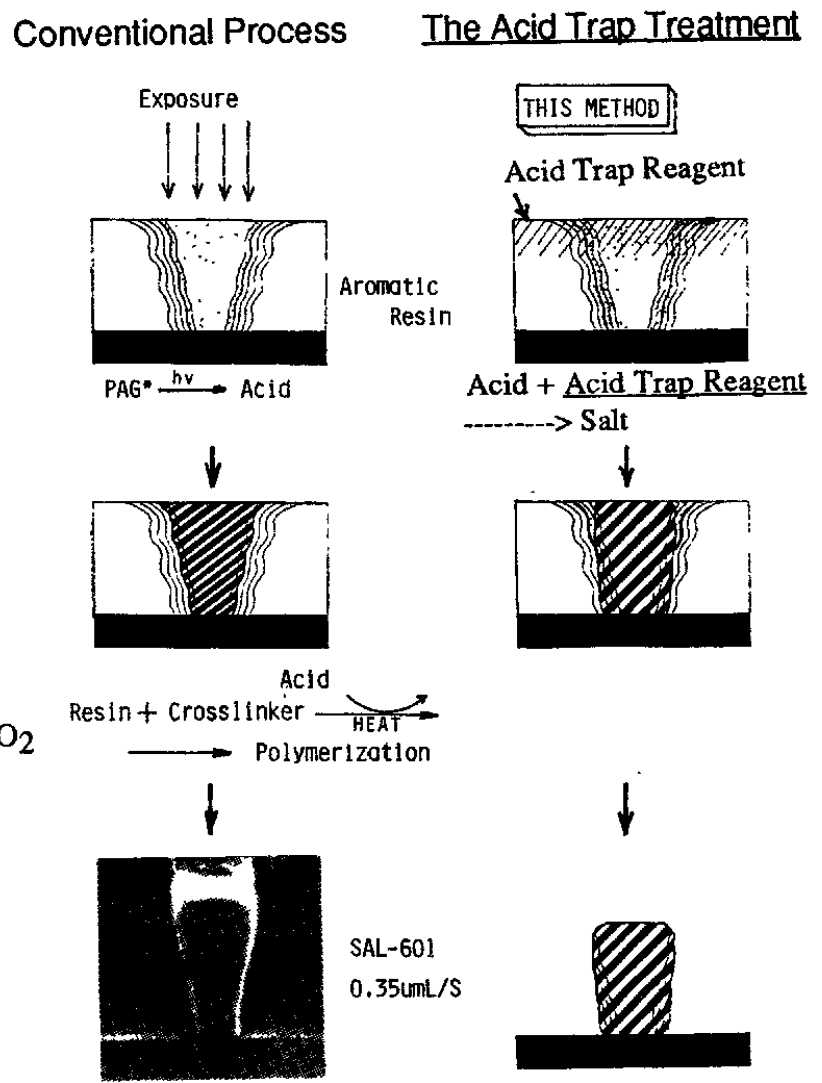

Fig-4. Concept of the acid trap treatment for chemically amplified negative resist 


\section{Result}

\section{Pattern characteristics}

Pattern profiles of lines and spaces obtained with the acid trap treatment of SAL601-ER7 are shown in Fig-9. These patterns are almost rectangular down to $0.3 \mu \mathrm{m}$. The re-entrant profiles of the conventional method were improved by using the acid trap treatment with imidazole.

\section{Evaluation of standing wave phenomenon}

Fig-10 shows $0.4 \mu \mathrm{m} \mathrm{C} / \mathrm{H}$ pattern shapes and a quantitative analysis of the standing wave phenomenon of the transparent chemically amplified photoresist XP8843. Two curves of C/H diameter vs. exposure energy show critical combinations of two resist thicknesses corresponding to maximum and minimum of sensitivity caused by standing wave phenomenon. In spite of XP8843's steep profiles the CD variations between these two states are very large. They range from $0.3 \mu \mathrm{m}$ to $0.5 \mu \mathrm{m}$.

On the other hand, highly absorbant chemically amplified resist SAL601-ER7 could dump the standing wave phenomenon (Fig-11). However, its profiles of $0.4 \mu \mathrm{m} \mathrm{C} / \mathrm{H}$ indicated typical reentrant shapes of high absorbant negative resist. Also it is difficult to apply the device fabrication process considering the dry etching process. It is understood that pattern profiles and standing wave phenomenon are trading off. This means, conventional target of excimer resists aiming at high transparency is not always suitable from the practical point of view.

Hence, the acid trap method was applied to $\mathrm{C} / \mathrm{H}$ patterning of SAL-601. The steep profiles as rectangular as XP8843's with dumping of the standing wave phenomenon were obtained (Fig-12). This result shows the possibility to control fractuation of $0.4 \mu \mathrm{m} \mathrm{C} / \mathrm{H}$ within $10 \%$ while maintaining practically affordable profiles.

\section{Application on topology}

Fig-13 shows an example of acid trap method applied to SAL601-ER7 on actual topological structure, the analog of TFT. Lines and spaces are $1.2 \mu \mathrm{m}$ pitch and step height is $2000 \AA .0 .4 \mu \mathrm{m}$ $\mathrm{C} / \mathrm{Hs}$ were obtained with excellent $\mathrm{CD}$ control both on the top and the bottom of steps. Thus we found the high potential of this method to satisfy both pattern profiles and standing wave dumping.

\section{Discussion}

\section{Characteristic curves with and without imidazole treatment}

Fig-14 shows characteristic curves of SAL601-ER7 with and without imidazole treatment. Although a slight degradation of sensitivity was observed, high contrast was kept.

\section{Dissolution rate depth profiles with and without imidazole treatment}

To investigate the mechanism of the acid trap treatment, the dissolution rate of the exposed and unexposed film was measured by DRM(Dissolution Rate Monitor). 
(1) Resist Coot

SAL601-ER7

$0.7 \mu \mathrm{mt}$ on $\mathrm{Si}$

Soft Baking

$90^{\circ} \mathrm{C} 60 \mathrm{sec}$

\section{Optimization of Imidazole 'Treatment}

\section{$0.4 \mu \mathrm{m}$ Contact Hole}

with KrF Excimer Laser Stepper

(2) Imidozole Treatment

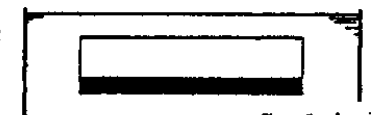

Soak in Imidazole sol.

I) Rinse with Water Mask $\downarrow \downarrow \downarrow \downarrow \downarrow \downarrow \downarrow \downarrow \downarrow \downarrow$

(3) Exposure

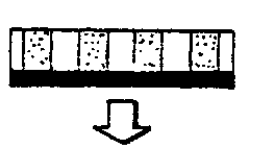

KrF Excimer NA 0.42

(4)PEB

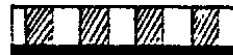<smiles>C1CCCC1</smiles>

$100^{\circ} \mathrm{C} 120 \mathrm{sec}$

(5) Development

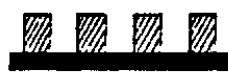

MF622(0.268N)

Dip.5min

(No Developmental Modification)

Fig-6. Process flow of the acid trap treatment

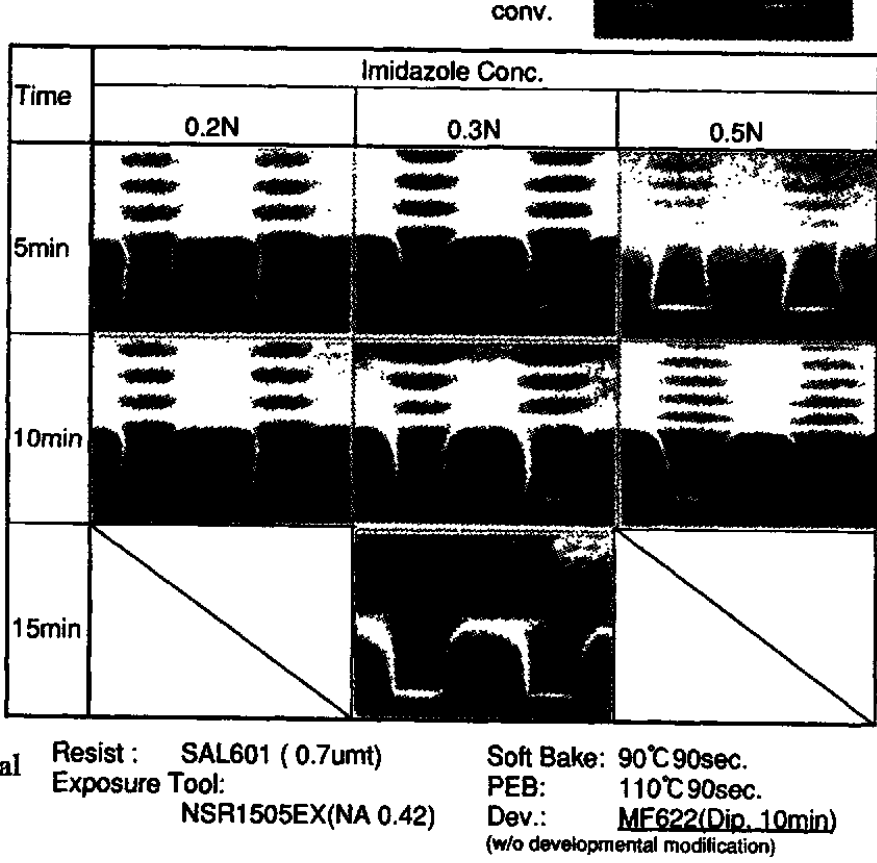

Fig-8. Optimization of imidazole soaking conditions; contact holes

OPTIMIZATION Of PROCESS CONDITION
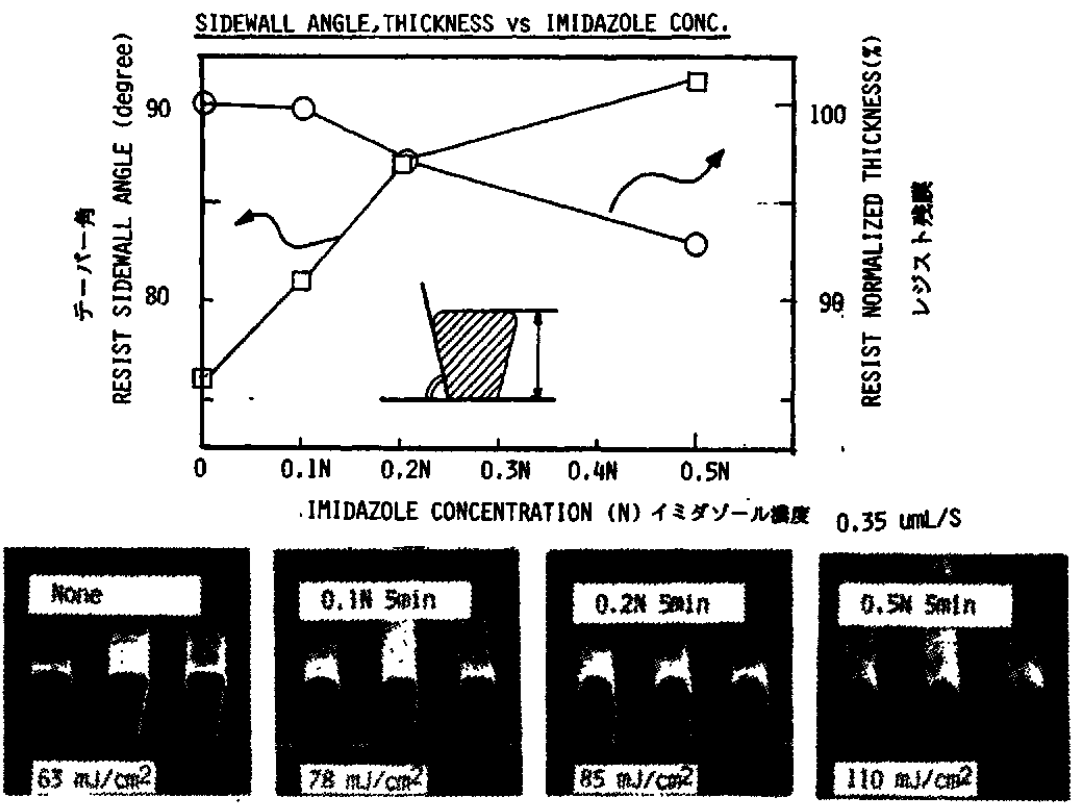

Fig-7. Optimization of imidazole soaking conditions; lines and spaces 
Pattern Profile Improvement by Imidozole Treatment
Conv.
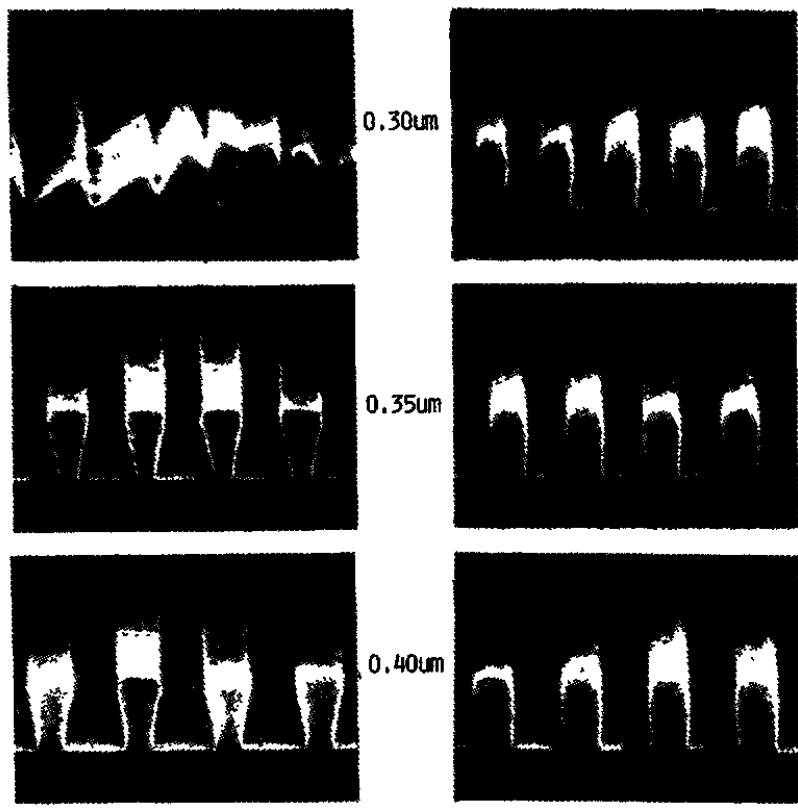

$62.5 \mathrm{~mJ} / \mathrm{cm}^{2}$
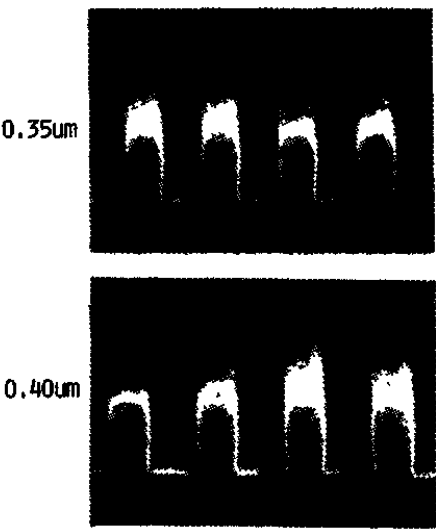

$85 \mathrm{~mJ} / \mathrm{cm}^{2}$

$\left(\begin{array}{l}\text { Soak in } 0.2 \mathrm{~N} \text { Imidazole Sol. } \\ \text { In } 5 \mathrm{~min} \text { before Exposure }\end{array}\right)$
Fig-9 Pattern profile improvement of SAL601 by the acid trap treatment; lines and spaces

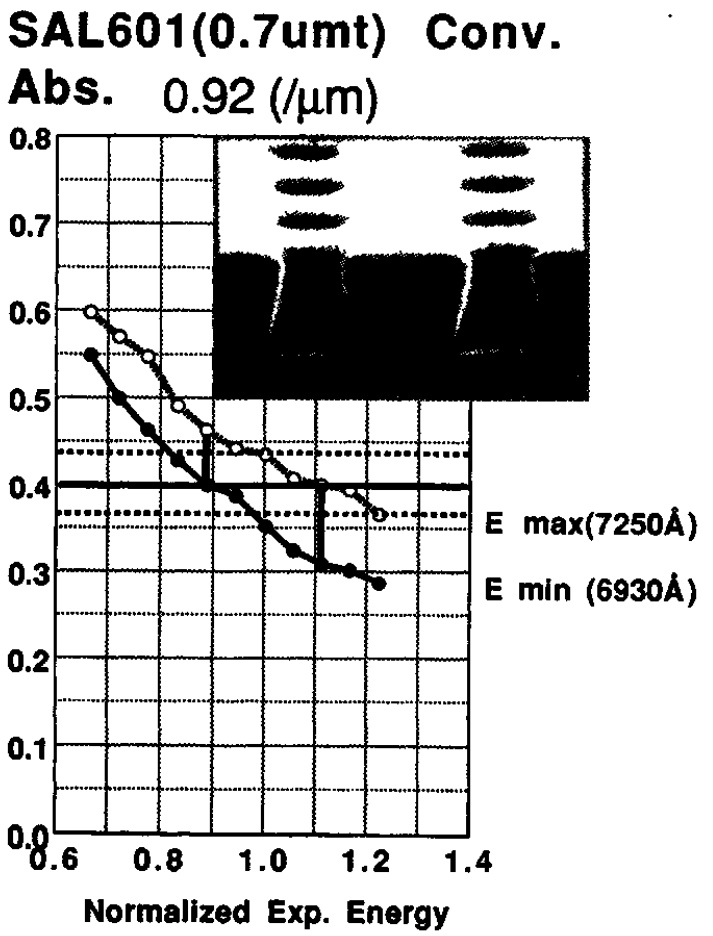

Fig-11. SAL601 $0.4 \mu \mathrm{m} \quad \mathrm{C} / \mathrm{H}$ pattern shapes Fig-12. SAL601 0.4 $\mu \mathrm{m} \mathrm{C} / \mathrm{H}$ pattem shapes with the acid (conventional) and evaluation of the standing wave phenomenon
XP8843 (0.7umt)

Abs. $0.24(/ \mu \mathrm{m})$

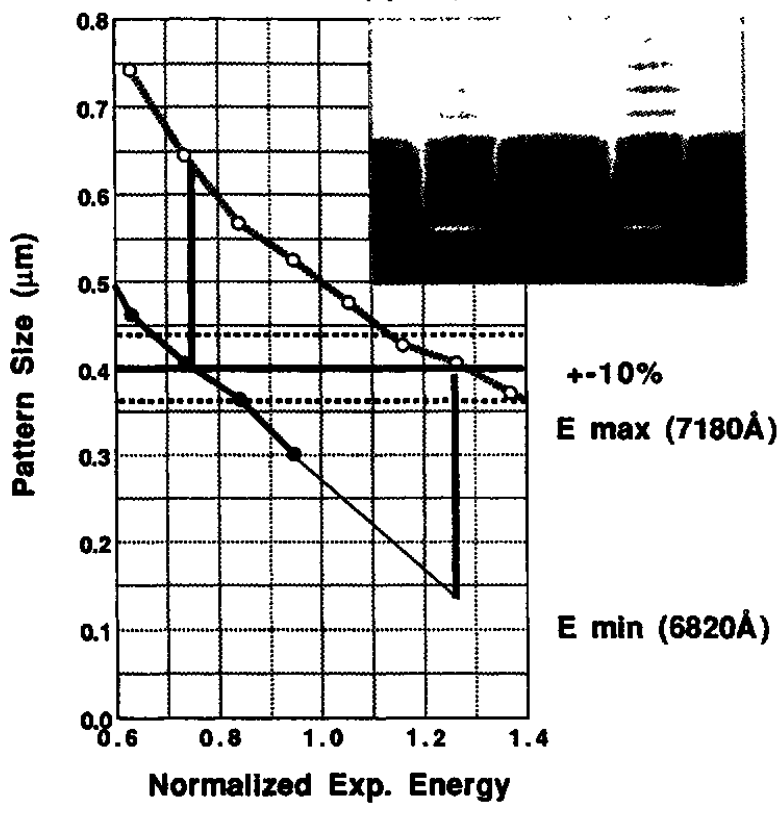

Fig-10. XP8843 $0.4 \mu \mathrm{m} \mathrm{C/H}$ pattern shapes and evaluation of the standing wave phenomenon; Two curves of $\mathrm{C} / \mathrm{H}$ diameter vs. exposure energy show critical combination of two resist thicknesses corresponding to maximum and minimum of sensitivity caused by standing wave phenomenon.

\begin{tabular}{l}
\hline SAL601 (0.7umt) \\
Imidazole Treatment
\end{tabular}

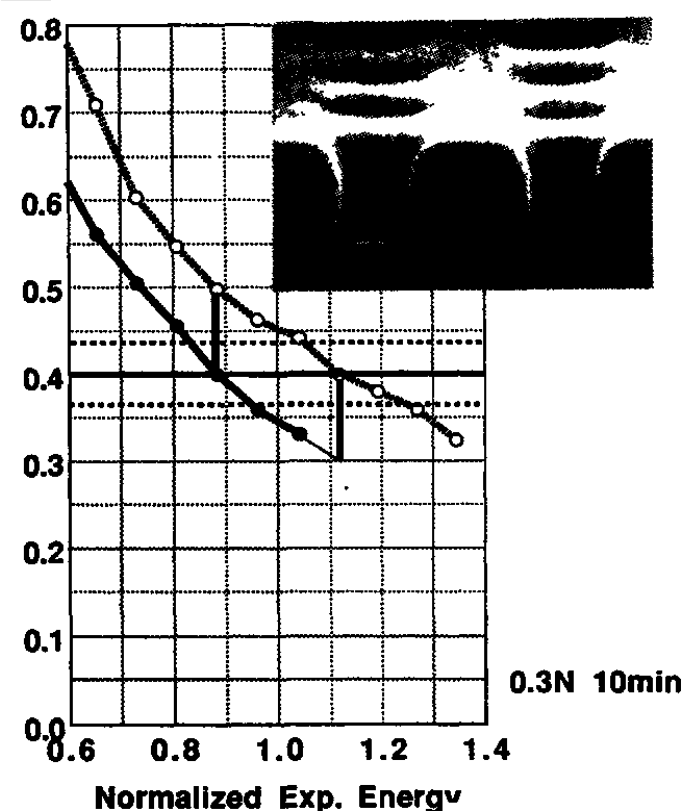
trap treatment and evaluation of the standing wave phenomenon 

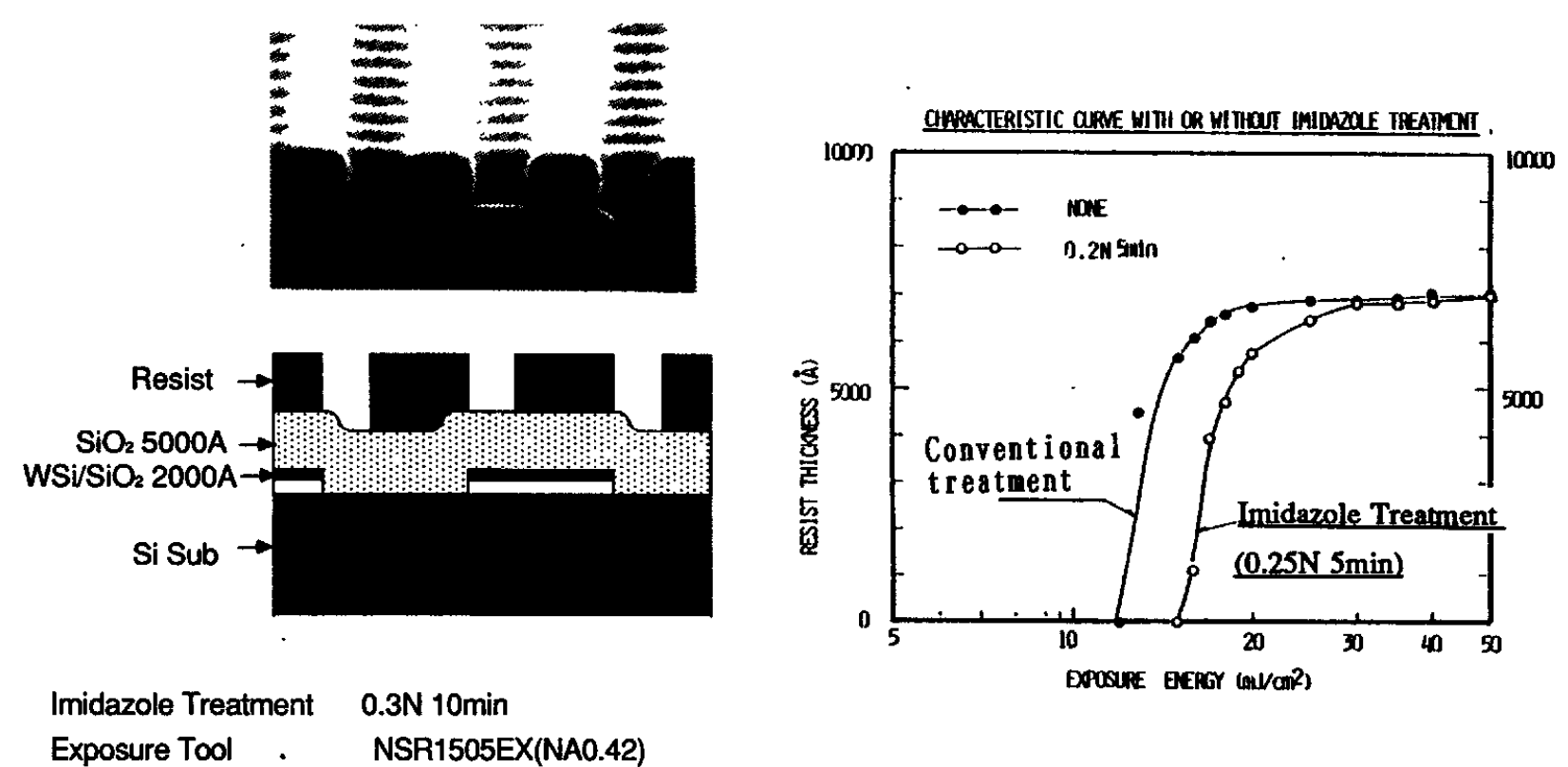

Fig-13. Application of the acid trap treatment to actual Fig-14. SAL601 characteristic curves with and without topology; $0.4 \mu \mathrm{m} \mathrm{C/H}$ imidazole treatment
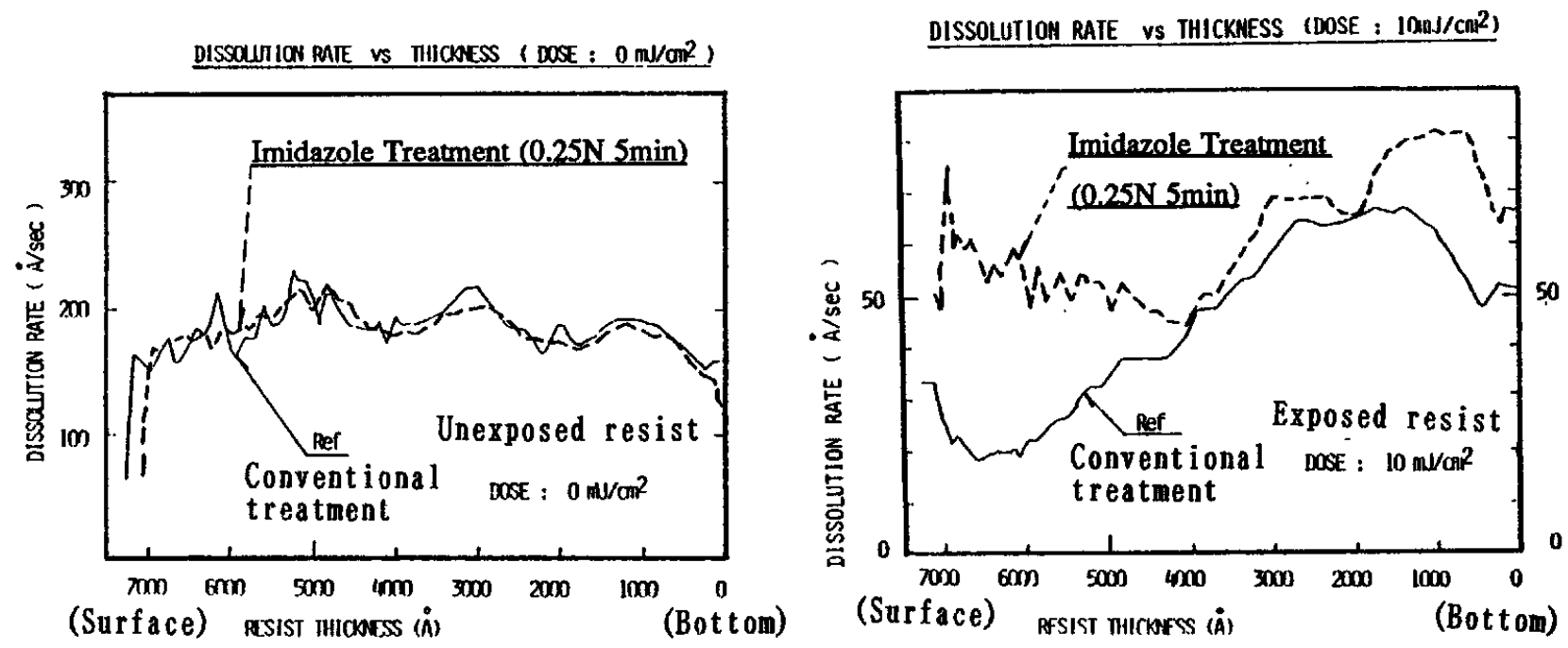

Fig-15. SAL601 dissolution rate depth profiles of Fig-16. SAL601 dissolution rate depth profiles of unexposed region with and without imidazole treatment exposed region $\left(10 \mathrm{~mJ} / \mathrm{cm}^{2}\right)$ with and without the imidazole treatment; Dissolution rate is constant from the top to bottom. Dissolution rate of resist surface was accelerated. 
Fig-15 shows the dissolution rates of unexposed region of SAL601-ER7 with and without imidazole treatment. Both dissolution rates are exactly the same from the resist top to the bottom. This indicates imidazole does not affect the resin itself.

On the other hand, in the exposed region we observed the great deference between this method and the conventional method. Dissolution rate curve without imidazole treatment is slopped from the top to the bottom corresponding to optical absorbance at $248 \mathrm{~nm}$ of SAL601-ER7 (Fig16). This tendency produced re-entrant photoresist profiles. On the contrary, with imidazole treatment the dissolution rate is almost constant from the top to the bottom along the resist thickness. The dissolution rate at the resist top is accelerated.

These results show that imidazole acts as an acid trap reagent only on the resist surface and yields dramatic pattern improvements of chemically amplified resist. The acid trap treatment affects directly the reactions of the resist pattern formation. This point is absolutely different from the conventional surface treatments for naphtoquinonediazide type resists such as alkaline treatment 5 ). In case of positive chemically amplified resist, this method might be applicable to the pattern profile improvement. The acid trap would act as surface inhibition by capturing excess amount of photogenerated acid on the resist surface.

\section{Conclusion}

1) A novel pattern profile improvement method, the acid trap treatment for chemically amplified negative photoresist utilizing the mechanism of acid catalyzed system is proposed. A dramatic pattern profile improvement was obtained.

2) From studying of dissolution rate depth profiles, we proved that imidazole acted as an acid trap reagent on the resist surface of the exposed region only.

3) The combination of this method and highly absorbant chemically amplified resist satisfied the requirements of both pattern profiles and control of $\mathrm{CD}$ variation.

\section{References}

1. J. M. J. Frechet, H. Ito, C. G. Willson, Microcircuit Eng., 2, 260(1982).

2. J. W. Thackeray, G. W. Orsula, E. K. Pavelcheck, D. Canistro, L. E. Bogan, Jr., A. K. Berry, K. A. Graziano, Proc. SPIE 1086-5(1989).

3. T. Kasuga, M. Saito, T. Tanaka, The Japan Society of Applied Physics, The 50th Fall Meeting, 28pL3(1989); T. Kasuga, T. Tanaka, Y. Tomo, The Japan Society of Applied Physics, The 51th Fall Meeting, 27pZG1(1990).

4. H. Y. Liu, M. P. deGrandpre, W. E. Feely, J. Vac. Sci. Tech. B., 6, 379(1988).

5. S. Ogawa, S. Uoya, H. Kimura, H. Nagata, J. Photopolym. Sci. Technol., Vol.2, No.3,375(1989). 